\title{
Regulated proteolysis of Trop2 drives epithelial hyperplasia and stem cell self-renewal via $\beta$-catenin signaling
}

\author{
Tanya Stoyanova, ${ }^{1}$ Andrew S. Goldstein, ${ }^{2,3,4}$ Houjian Cai, ${ }^{1,7}$ Justin M. Drake, ${ }^{1}$ Jiaoti Huang, ${ }^{5}$ \\ and Owen N. Witte 1,2,3,4,6,8 $^{1,2}$ \\ ${ }^{1}$ Department of Microbiology, Immunology, and Molecular Genetics, University of California at Los Angeles, Los Angeles, \\ California 90095, USA; ${ }^{2}$ Department of Molecular and Medical Pharmacology, University of California at Los Angeles, Los \\ Angeles, California 90095, USA; ${ }^{3}$ Jonsson Comprehensive Cancer Center, University of California at Los Angeles, Los Angeles, \\ California 90095, USA; ${ }^{4}$ Eli and Edythe Broad Center of Regenerative Medicine and Stem Cell Research, University of California \\ at Los Angeles, Los Angeles, California 90095, USA; ${ }^{5}$ Department of Pathology and Laboratory Medicine, University of \\ California at Los Angeles, Los Angeles, California 90095, USA; ${ }^{6}$ Howard Hughes Medical Institute, University of California at \\ Los Angeles, Los Angeles, California 90095, USA
}

The cell surface protein Trop2 is expressed on immature stem/progenitor-like cells and is overexpressed in many epithelial cancers. However the biological function of Trop2 in tissue maintenance and tumorigenesis remains unclear. In this study, we demonstrate that Trop2 is a regulator of self-renewal, proliferation, and transformation. Trop2 controls these processes through a mechanism of regulated intramembrane proteolysis that leads to cleavage of Trop2, creating two products: the extracellular domain and the intracellular domain. The intracellular domain of Trop2 is released from the membrane and accumulates in the nucleus. Heightened expression of the Trop2 intracellular domain promotes stem/progenitor self-renewal through signaling via $\beta$-catenin and is sufficient to initiate precursor lesions to prostate cancer in vivo. Importantly, we demonstrate that loss of 及-catenin or Trop2 loss-of-function cleavage mutants abrogates Trop2-driven self-renewal and hyperplasia in the prostate. These findings suggest that heightened expression of Trop2 is selected for in epithelial cancers to enhance the stem-like properties of self-renewal and proliferation. Defining the mechanism of Trop2 function in self-renewal and transformation is essential to identify new therapeutic strategies to block Trop2 activation in cancer.

[Keywords: Trop2; self-renewal; transformation; regulated intramembrane proteolysis; $\beta$-catenin]

Supplemental material is available for this article.

Received May 15, 2012; revised version accepted September 6, 2012.

Trop2 is a type I transmembrane glycoprotein highly expressed in various epithelial cancers, such as advanced squamous cell carcinoma of the oral cavity and colorectal, pancreatic, gastric, and ovarian cancer, and its high levels correlate with poor prognosis and survival (Nakashima et al. 2004; Ohmachi et al. 2006; Fong et al. 2008a,b; Kobel et al. 2008; Fang et al. 2009; Muhlmann et al. 2009). Monoclonal antibodies targeting Trop2 exhibit potent anti-cancer activity through cytotoxicity in multiple xenograft models of cancer, including prostate, pancreatic, breast, colon, endometrial, and lung cancer (Young et al. 2008; Govindan et al. 2009; Alberti 2012).

${ }^{7}$ Present address: Hollings Cancer Center, Department of Medicine, Medical University of South Carolina, Charleston, SC 29425, USA.

${ }^{8}$ Corresponding author

E-mail owenwitte@mednet.ucla.edu

Article is online at http://www.genesdev.org/cgi/doi/10.1101/gad.196451.112. Freely available online through the Genes \& Development Open Access option.
Knockdown of Trop2 abrogates the growth of colon cancer cell lines and inhibits tumor initiation and progression in mice (Wang et al. 2008; Trerotola et al. 2012a). Recently, Trop2 was demonstrated to play dual functions in tumorigenesis-as a tumor suppressor and an oncogene (Wang et al. 2008, 2011).

Cancer cells share multiple characteristics with adult tissue stem cells, such as self-renewal and proliferative capacity. Many factors that regulate stem/progenitor function and development have been found altered in prostate cancer. Activation of the PI3K pathway promotes self-renewal and has a prominent role in prostate malignancy (Yoshimoto et al. 2006; Mulholland et al. 2009). The Polycomb protein Bmi-1 regulates both selfrenewal and tumorigenesis in the murine prostate, and its increased expression correlates with unfavorable outcome (van Leenders et al. 2007; Lukacs et al. 2010). Other pathways, such as Wnt and Notch, control the balance between stem/progenitor self-renewal and differentiation 
and are also frequently dysregulated in prostate cancer (Shou et al. 2001; Chen et al. 2004; Wang et al. 2006).

Several groups have demonstrated that Trop2 is a marker of stem/progenitor cells in adult tissues. Trop2 is not expressed in the normal liver but appears on the undifferentiated oval cells shortly after their activation due to liver injury (Okabe et al. 2009). Trop2 also enriches for endometrial-regenerating cells in a dissociated cell tissue recombination assay (Memarzadeh et al. 2010). In the human and mouse prostate, the Trop2-expressing subpopulation of basal cells (Trop2 ${ }^{\text {hi }}$ ) possesses stem cell capacities such as self-renewal, tissue regeneration, and multilineage differentiation (Goldstein et al. 2008, 2010).

Despite its broad expression in cancer, little is known about Trop2-mediated signaling. A recent study demonstrated that Trop 2 inhibits cell adhesion by promoting the interaction between $\beta-1$ integrin and RACK 1 in prostate cancer cell lines (Trerotola et al. 2012b). Trop2 has been reported to control the interaction with the extracellular matrix during kidney development (Tsukahara et al. 2011). Finally, Trop2 is demonstrated to regulate $\mathrm{Ca}^{2+}$ signaling, and its cytoplasmic tail is phosphorylated by protein kinase C (PKC) in vitro (Basu et al. 1995; Ripani et al. 1998). The downstream signals transmitted upon phosphorylation of Trop2 remain to be defined.

Regulated intramembrane proteolysis (RIP) is a mechanism of processing and activation of transmembrane proteins, including adhesion molecules such as $\mathrm{N}$-cadherin and E-cadherin (Brown et al. 2000; Maretzky et al. 2005; Lal and Caplan 2011; Solanas et al. 2011). Members of the Notch family of receptors, involved in cell fate decisions, are also regulated by RIP (De Strooper et al. 1999). RIP has been recently associated with cancer. Activating mutations of Notch 1 resulting in its cleavage are found in $56 \%$ of patients suffering from T-ALL (T-cell acute lymphoblastic leukemia) and are also associated with various solid tumors (Weng et al. 2004; Lobry et al. 2011; Ranganathan et al. 2011). A recent study revealed that EpCAM, an adhesion protein that shares 50\% homology with Trop2, functions in cellular transformation via RIP cleavage mechanisms (Maetzel et al. 2009). High levels of activated EpCAM are detected in breast, prostate, head and neck, and esophageal cancers and has been associated with poor prognosis in thyroid cancer (Ralhan et al. 2010a,b).

Although Trop2 expression is elevated in various carcinomas and serves as a marker of stem/progenitor cells, the functional role of Trop2 in self-renewal, its relation to transformation, and the molecular mechanisms by which Trop2 transmits signals to regulate these processes remain unclear. In the present study, we demonstrate that Trop2 controls stem/progenitor self-renewal and tissue regeneration in the prostate. Trop2 is activated by RIP, and its cleavage is carried out by the TNF- $\alpha$-converting enzyme (TACE) followed by $\gamma$-secretase cleavage within the transmembrane domain, resulting in shedding of the extracellular domain (ECD) and accumulation of the intracellular domain (ICD) to the nucleus. Nuclear ICD is found in human prostate cancer but not in the cancer- adjacent benign tissue, suggesting a role for Trop2 cleavage in tumorigenesis. The ICD alone promotes self-renewal, initiates prostatic intraepithelial neoplasia (PIN), and is involved in a signaling cascade dependent on $\beta$-catenin. Nuclear $\beta$-catenin colocalizes with nuclear ICD in human prostate cancer. Moreover, loss of $\beta$-catenin abolishes Trop2-driven self-renewal and transformation activity. Blocking cleavage of Trop2 by mutating its cleavage sites abrogates its self-renewal and transformation capacity, demonstrating that RIP is required for Trop2 activity. Defining the functional role of Trop2 in self-renewal and transformation and delineating the molecular mechanism of Trop2 action may promote development of new therapeutic strategies to inhibit Trop2 signaling in a wide range of epithelial tumors.

\section{Results}

Trop2 regulates self-renewal of stem/progenitor cells and tissue regeneration in the adult prostate

Stem/progenitor cells can be expanded in an in vitro sphere assay in which sphere number over multiple passages measures self-renewal activity, and sphere size is a reflection of progenitor proliferation capacity (Reynolds and Weiss 1996; Dontu et al. 2003a,b, 2004; Xin et al. 2007; Lukacs et al. 2010). By using cell surface markers CD49f, Sca-1, and Trop2, we previously demonstrated the ability to enrich for luminal $\left(\mathrm{CD} 49 \mathrm{f}^{\text {lo }}, \mathrm{Sca}^{-} \mathrm{1}^{-}\right)$and basal (LSC or CD49f $\left.{ }^{\text {hi }}, \mathrm{Scal}^{+}\right)$cells and to further subdivide the basal cells into Trop $2^{10}\left(\mathrm{LSCT}^{\mathrm{lo}}\right)$ and stem/progenitor enriched Trop $2^{\text {hi }}$ fractions (LSCT ${ }^{\text {hi }}$ ) (Supplemental Fig. S1A; Lawson et al. 2007; Goldstein et al. 2008). To investigate the functional role of Trop2 in stem/progenitor cell self-renewal, we constructed lentiviral vectors expressing a potent Trop2 microRNA (miRNA) mimic (miRNA Trop2) to diminish the levels of Trop2 or a nontargeting scrambled miRNA (miRNA scrambled) carrying the green fluorescent protein (GFP) for visualization of transduced cells (Supplemental Fig. S1B). The progenitor-enriched population LSCT ${ }^{\text {hi }}$ was transduced with miRNA Trop2 or miRNA scrambled, plated in the sphere assay, and further passaged to second-generation spheres to measure selfrenewal (Gen 2) (Fig. 1A). Trop2 knockdown resulted in a fourfold reduction in self-renewal activity, as measured by sphere number upon passaging (Gen 2) (Fig. 1A). The progenitor proliferation capacity assessed by the sphere size was also significantly reduced upon knockdown of Trop2, as demonstrated by the twofold reduction in sphere diameter (Fig. 1A).

Dissociated prostate cells are able to regenerate prostatic tubules when combined with urogenital sinus mesenchyme (UGSM) and implanted under the kidney capsule of severe combined immunodeficiency (SCID) mice (Cunha and Lung 1978; Xin et al. 2003). Previous interrogation of different epithelial subpopulations revealed that only LSCT $^{\text {hi }}$ basal stem/progenitor cells possess regenerative activity and can give rise to a prostate-like structure in the in vivo regeneration assay (Goldstein et al. 2008). To assess the role of Trop2 in prostate tubule formation and 


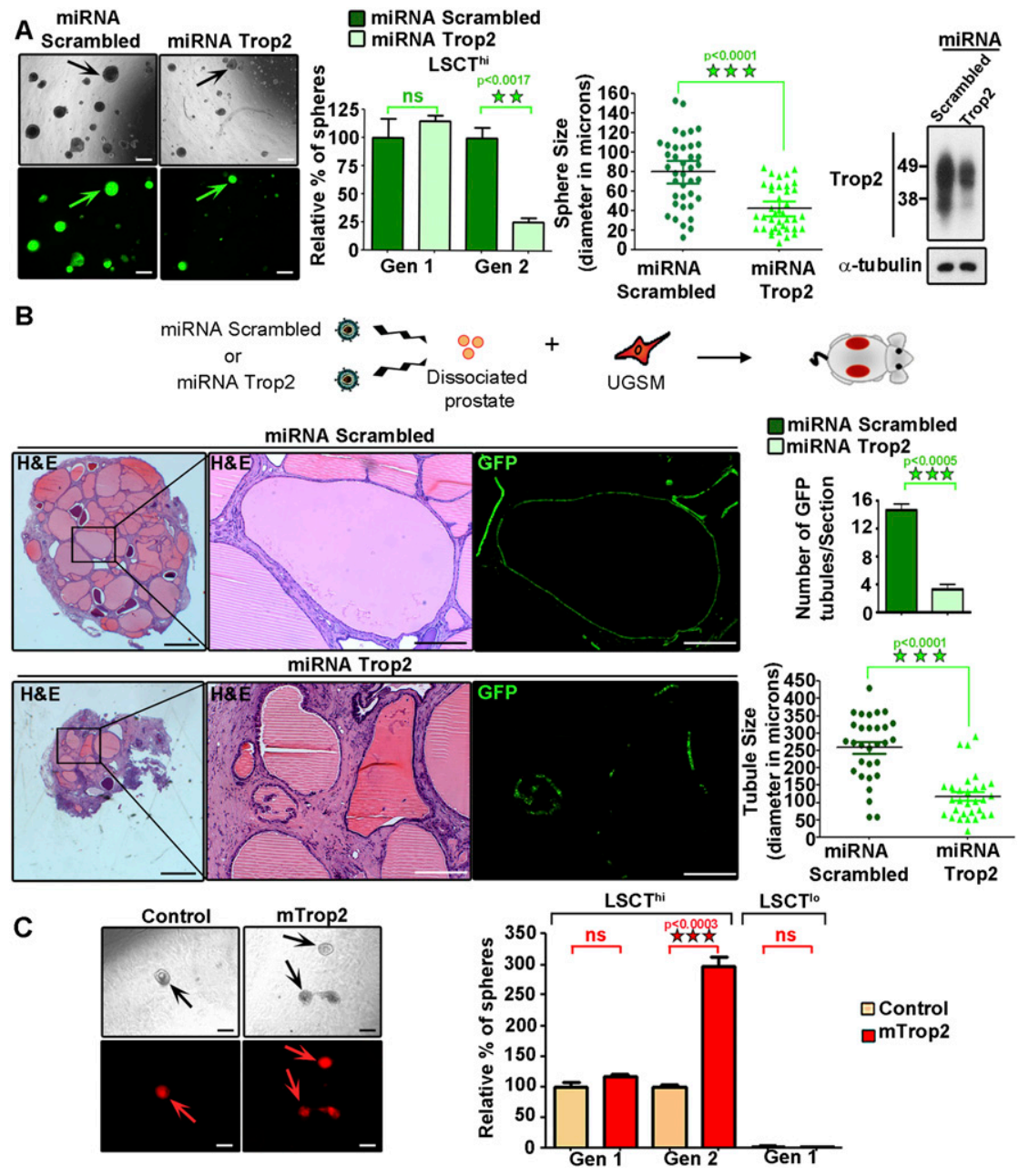

Figure 1. Trop2 regulates self-renewal in the prostate. $(A)$ Prostate progenitor cells $\left(\mathrm{LSCT}^{\mathrm{hi}}\right.$ ) were isolated by fluorescence-activated cell sorting (FACS). An equal number of LSCT ${ }^{\text {hi }}$ cells was infected with a lentivirus expressing scrambled miRNA and GFP (miRNA Scrambled) or a lentivirus expressing Trop2 miRNA and GFP (miRNA Trop2), followed by the in vitro sphere assay. Each sample in the experiment was plated in triplicate. Three independent experiments were performed. The left panels show representative sphere pictures and nearly $100 \%$ infection efficiency, assessed by GFP-positive spheres. Bar, $100 \mu \mathrm{m}$. Sphere number and diameter, referred to as sphere size in microns, were counted at generation 1 (Gen 1). Spheres were dissociated into single cells, and an equal number of cells was plated for passaging to generation 2 (Gen 2). Sphere number is presented as percentage normalized to miRNA Scrambled. Data are represented as mean of the triplicates \pm SEM. Statistical analysis is shown. (ns) Nonsignificant. The level of Trop2 knockdown is shown by Western blot in spheres at Gen 1 prior to replating for Gen 2. Trop2 is highly glycosylated and appears as an irregular band between 35 and $50 \mathrm{kD}$ (Supplemental Fig. S1C). (B) Trop2 expression was downregulated in dissociated primary prostate cells via transduction with miRNA Scrambled or miRNA Trop2 lentivirus expressing GFP followed by the in vivo regeneration assay. One out of three representative recovered grafts for each condition is shown. Bars: left panels, $800 \mu \mathrm{m}$; right panels, $100 \mu \mathrm{m}$. The number of GFP tubules per section for

each graft was counted in miRNA Scrambled or miRNA Trop2 grafts and plotted as mean \pm SEM. Diameter of 30 GFP-positive tubules was measured in microns. $(C)$ An equal number of LSCT ${ }^{\text {hi }}$ or LSCT ${ }^{\text {lo }}$ cells was transduced with a RFP-expressing (control) or mouse Trop2- and RFP-expressing (mTrop2) lentivirus and plated in triplicate for each sample in an in vitro sphere assay. Three independent experiments were performed. The left panels represent spheres from LSCT ${ }^{\text {hi }}$ transduced with either RFP (control) or mTrop2 and RFP (mTrop2). Bars, $100 \mu \mathrm{m}$. LSCT ${ }^{\text {hi }}$ sphere number was counted at Gen 1 and Gen 2, while LSCT ${ }^{\text {lo }}$ sphere number was counted only at Gen 1, since no growth was observed. Sphere number is presented as percentage normalized to RFP transduced spheres and presented as mean of the triplicates \pm SEM.

regeneration in vivo, dissociated primary prostate cells $\left(2.5 \times 10^{5}\right)$ were transduced with miRNA Trop2 or miRNA scrambled lentivirus at an equivalent multiplicity of infection (MOI = 50) (Fig. 1B). Transduced prostate cells were combined with UGSM cells $\left(2.5 \times 10^{5}\right)$ and subjected to an in vivo regeneration assay (Fig. 1B). Upon histological analysis of the recovered grafts, we observed that Trop2 knockdown grafts contained fourfold fewer infected tubules when compared with the control (Fig. 1B). Tubules derived from Trop2 knockdown epithelium were also smaller, with an average diameter of $100 \mu \mathrm{m}$, in contrast to the control grafts, with an average diameter of $260 \mu \mathrm{m}$ (Fig. 1B). Trop2 loss attenuates prostate regeneration in vivo and sphere formation in vitro, demonstrating that Trop2 not only is a marker for stem/progenitor cells, but also functionally regulates adult tissue selfrenewal and prostate regeneration.
The functional role of Trop2 in self-renewal was also assessed by measuring sphere formation upon Trop2 overexpression. LSCT $^{\text {lo }}$ and $\mathrm{LSCT}^{\mathrm{hi}}$ basal cells were infected with either mouse Trop2- and RFP-expressing (mTrop2) or RFP-expressing (Control) lentivirus (Fig. 1C; Supplemental Fig. S1D). Heightened Trop2 promoted a threefold expansion of Gen 2 cells (Fig. 1C). Consistent with our previous report, $\mathrm{LSCT}^{\text {lo }}$ cells lack sphere-forming activity (Goldstein et al. 2008). Ectopic expression of Trop 2 was not sufficient to induce sphere growth in $\mathrm{LSCT}^{\text {lo }}$ cells (Fig. $1 \mathrm{C}$ ), suggesting that Trop2 ${ }^{\text {lo }}$ cells may lack signaling components important for Trop2 activity. Previous studies demonstrating that $\beta$-catenin promotes the expansion of prostate spheres (Lukacs et al. 2010; Shahi et al. 2011) led us to test the status of $\beta$-catenin in LSCT ${ }^{\text {lo }}$ versus LSCT ${ }^{\text {hi }}$ basal cells. Confocal microscopy revealed significantly lower levels 
of $\beta$-catenin in LSCT $^{\text {lo }}$ in comparison with LSCT $^{\text {hi }}$ (Supplemental Fig. S2).

Trop2 is processed by proteolytic cleavage, generating the ICD and ECD

We noted Trop2 localization in the nucleus in a small fraction of cells. To quantitatively measure Trop2 localization, a mouse prostate epithelial basal (PEB) cell line (Salm et al. 2000) that expresses endogenous Trop2 was used. PEB cells were infected with lentivirus expressing RFP or RFP and Trop2 modified with a C-terminal Myc tag to follow Trop2 ICD. Confocal microscopy revealed that the ICD is found on the membrane in $100 \%$ of the cells and within the nucleus in $15 \%$ of the cells, while Trop2 ECD can be detected only on the plasma membrane and in the cytoplasm (Fig. 2A). We performed further analysis to determine whether Trop2 ECD is shed from the cellular membrane in PEB and another prostate epithelial cell line, TRAMP-C2, cells (Foster et al. 1997). Trop2 ECD, but not ICD, was detected in precipitated medium from cells expressing Trop2 (Fig. 2B). Taken together, these data support a model where Trop2 is cleaved, resulting in shedding of the ECD and accumulation of the ICD in the nucleus (Fig. 2A,B).

Trop2 cleavage products independently stimulate self-renewal and proliferation

Given that Trop2 is cleaved, releasing two fragments (ECD and ICD), we asked whether these different domains serve alternative functional roles in the prostate. Lentivirus carrying either the ICD or the secreted ECD fused to the Fc region of human IgG1 to ensure proper secretion and stability (Trop2-ECD-Fc fusion) was generated (Supplemental Fig. S1D). ICD expression is demonstrated by immunofluorescence (Supplemental Fig. S3A). Dissociated primary mouse prostate cells were infected with either control lentivirus expressing RFP (control) or lentivirus expressing mouse Trop2 ICD and RFP (mICD) and were plated in the sphere assay. The ICD was sufficient to increase sphere formation and stem/progenitor proliferation, measured by sphere number and size, even prior to replating in Gen 1, suggesting that the ICD is the functionally dominant portion of the molecule (Fig. 2C). Further passaging showed continued enhancement of self-renewal activity, as measured by sphere number in Gen 2 (Fig. 2C).

Next, we tested the role of the ECD in self-renewal and proliferation. 293T cell lines were transduced with either a control lentivirus expressing RFP or a lentivirus expressing both ECD-Fc and RFP to generate secreted ECD that we confirmed by Western blot (Fig. 2D). LSCT $^{\text {hi }}$ cells were plated in the sphere assay and treated with either conditioned medium from the control 293T (CM) or conditioned medium containing ECD-Fc (CM+ECD) (Fig. 2D). Secreted ECD caused an increase in sphere size but not in sphere number, suggesting that the ECD increases the proliferation of prostate stem/progenitor cells (Fig. 2D). The activation of RIP is induced by ligand binding to its receptor (Schroeter et al. 1998; Mumm et al. 2000). Trop2 is an orphan receptor without a known ligand. We investigated the effects of the ECD on Trop2 processing. Upon treatment of prostate cells with secreted ECD by $293 \mathrm{~T}$ cells, we observed the appearance of small-molecular-weight fragments at a size of $6 \mathrm{kD}$, suggesting that Trop2 is cleaved (Supplemental Fig. S3B). Further studies will be necessary to rule out whether the ECD induces Trop2 cleavage by direct homophilic interaction or through distinct binding partners.

\section{Trop2 is cleaved by RIP}

Detection of the ECD and ICD at different cellular compartments and their independent function in self-renewal and proliferation led us to investigate the mechanisms through which Trop2 is being cleaved. TACE is a member of the ADAM family of proteases that mediates the initial proteolysis and ectodomain shedding of several transmembrane proteins during RIP, followed by intramembrane proteolysis carried out by the $\gamma$-secretase complex. To test whether TACE and $\gamma$-secretase play a role in Trop2 processing, PEB cells expressing Trop2-Myc tag were treated with the TACE inhibitor (TAPI-2) or $\gamma$-secretase inhibitor (DAPT). Treatment with TAPI-2 resulted in a significant increase in the levels of uncleaved full-length Trop2 (Fig. 3A; Supplemental Fig. S4A). Treatment of PEB cells with DAPT caused a significant increase in the fulllength Trop2 as well as the appearance of an intermediate cleavage product (ICP) (Fig. 3A, Supplemental Fig. S4A). The ICP of $\sim 15 \mathrm{kD}$ in size can be generated if Trop2 is not fully processed, but the first TACE cut still takes place (Fig. 3A). Treatment with DAPT and TAPI-2 also resulted in significant decrease of ICD localized in the nucleus (Fig. 3B). While RIP has been implicated in the activation of several transmembrane proteins, for the first time, we demonstrate that Trop 2 is cleaved and its processing involves RIP.

Presenilin 1 (PS-1) and PS-2 are catalytic subunits of the $\gamma$-secretase complex. Full-length Trop2 increases upon silencing of PS-1 and to a lesser extent upon silencing of PS-2 by siRNA (Fig. 3C; Supplemental Fig. S4B,C). Upon knockdown of either PS-1 or PS-2, we observed accumulation of Trop2 ICP similar to DAPT treatment (Fig. 3C). Knockdown of both PS-1 and PS-2 has an additive effect on Trop2 processing, as we observed a greater accumulation of ICP (Fig. 3C). An increase in Trop2 on the cell surface was detected upon PS-1 knockdown and to a lesser extent upon PS-2 knockdown, as measured by fluorescence-activated cell sorting (FACS) analysis (Supplemental Fig. S4C). These observations suggest that PS-1 is the major protease involved in Trop2 processing.

\section{Trop2 and EpCAM have distinct expression patterns in the adult mouse prostate}

Related proteins can often carry out different functions in distinct cell populations. Since we found that Trop2 is processed through RIP, we investigated the expression patterns of Trop2 and EpCAM, a related molecule previously demonstrated to be regulated by RIP (Maetzel 
\%
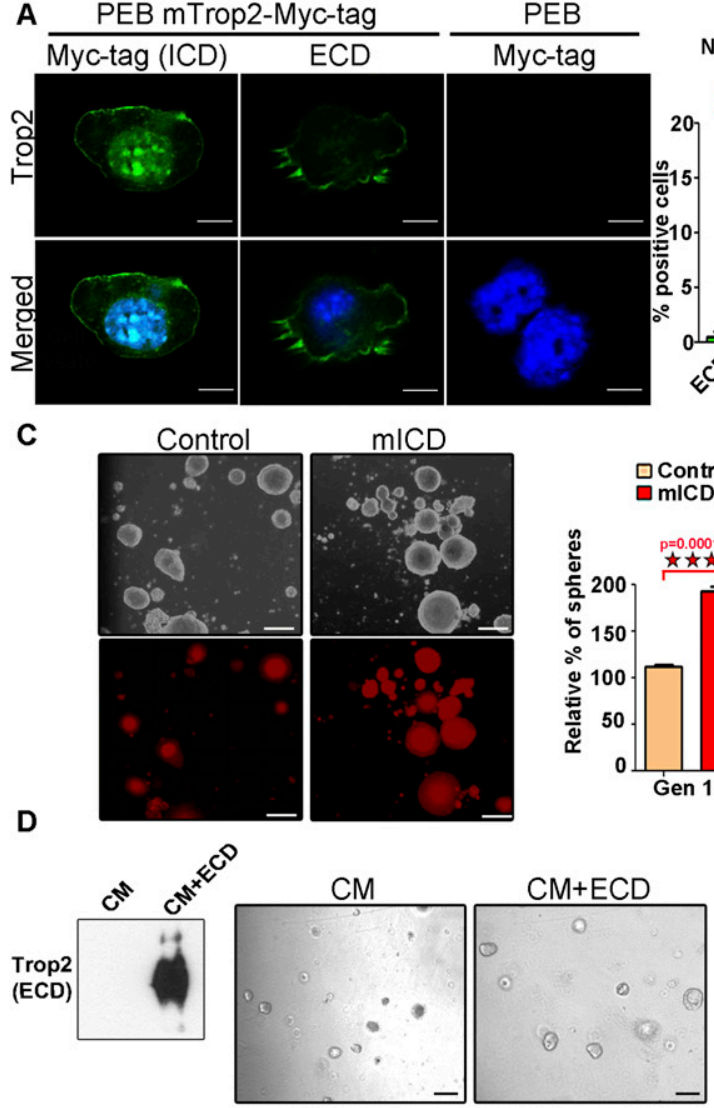

PEB Myc-tag
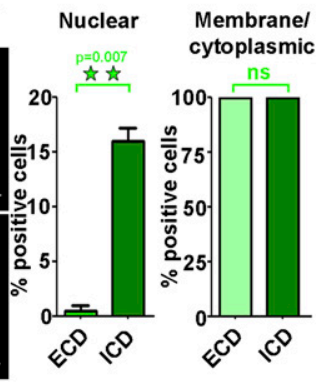

B

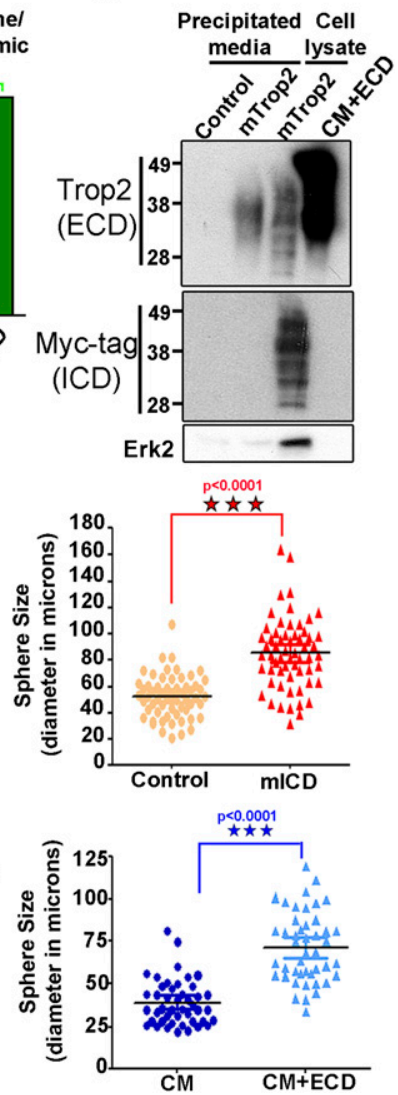

Figure 2. Trop2 ECD and ICD localize at distinct sites within the cell. Trop2 regulates stem/progenitor self-renewal through ICD and ECD cleavage products. (A) Confocal microscopy of PEB cells stained with anti-Myc tag or anti-ECD antibody and DAPI. The right two panels represent PEB cells infected with lentivirus expressing RFP (PEB) as a negative control to determine whether anti-Myc tag antibody recognizes any endogenous myc. Staining of PEB cells expressing mTrop2 with a C-terminal Myc tag to follow the ICD with the indicated antibodies is presented in the left four panels. Bar, $10 \mu \mathrm{m}$. One out of three independent experiments is shown. Graphs represent the percentage of cells with nuclear ICD or ECD (left graph) and cytoplasmic and membrane ICD or ECD (right graph). (B) Serum-free medium from TRAMP-C2 cells transduced with RFP lentivirus (control) or mTrop2-Myc tag (mTrop2) was precipitated (precipitated media), followed by Western blot using commercial anti-ECD antibody or anti-Myc tag recognizing the ICD or Erk2 as a marker for cell lysate. Cell lysates from TRAMP-C2 cells expressing mTrop2-Myc tag (mTrop2) and CM produced by $293 \mathrm{~T}$ expressing secreted Trop2-ECD-Fc (CM+ECD) were used as positive controls. Only the ECD of Trop2 was detected in the precipitated media. Highly glycosylated Trop2 is shown between 35 and $50 \mathrm{kD}$. Four independent experiments were performed. (C) An equal number progenitor cells (LSCT ${ }^{\text {hi }}$ ) was transduced with a control RFP-expressing (control) or Trop2 ICD-expressing (mICD) lentivirus and plated in triplicates in the sphere assay. Representative pictures are presented in the left panels. Bars, $100 \mu \mathrm{m}$. Seven days later, we observed an increase in sphere size and number as early as Gen 1 (graphs presented on the right). Orange panels represent RFP-infected spheres (control), and red panels show mICD-infected spheres. The ICD alone enhance self-renewal, as measured by sphere number in Gen 2. Sphere number in each sample is presented as the percentage normalized to the RFP-infected spheres (control). Data are represented as mean \pm SEM. (Right graph) Sphere size is presented in diameter in microns. Three independent experiments were performed. $(D)$ Generation of 293T cells line expressing secreted ECD. (Left) Conditioned medium produced by 293T expressing RFP (CM) or Trop2ECD-Fc and RFP (CM+ECD) was subjected to Western blot analysis with anti-ECD antibody. Equal numbers of progenitor cells $\left(\mathrm{LSCT}^{\mathrm{hi}}\right)$ were plated in triplicate and treated with conditioned medium from control 293T cells infected with RFP (CM) or 293T cells stably expressing and secreting Trop2-ECD-Fc and RFP (CM+ECD). Images show spheres grown in the presence of CM or CM+ECD. Bars, $100 \mu \mathrm{m}$. No difference in sphere number was observed (left graph), while there was a significant increase in sphere size upon treatment with CM+ECD at Gen 1 (right graph). Dark-blue bars represent spheres treated with CM, and light-blue bars represents spheres treated with $\mathrm{CM}+\mathrm{ECD}$. Three independent experiments were performed.

et al. 2009). The spatial patterns of Trop2 and EpCAM in the mouse prostate revealed dramatic differences in their cellular localization. While Trop2 expression was restricted to the progenitor-enriched region most proximal to the urethra, as previously reported (Goldstein et al. 2008), EpCAM was expressed evenly in all epithelial cells
(Supplemental Fig. S5A). FACS analysis demonstrated that Trop2 is expressed primarily in a subpopulation of Sca- $1^{+} \mathrm{CD} 49 \mathrm{f}^{\text {hi }}$ basal cells and is rarely expressed on luminal cells, while EpCAM was expressed in all CD49f ${ }^{\text {lo }}$ luminal and CD49fhi basal epithelial cells (Supplemental Fig. S5B). We hypothesize that EpCAM and Trop2 regulate 
A



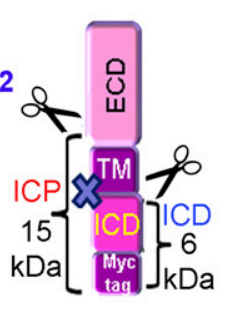

B

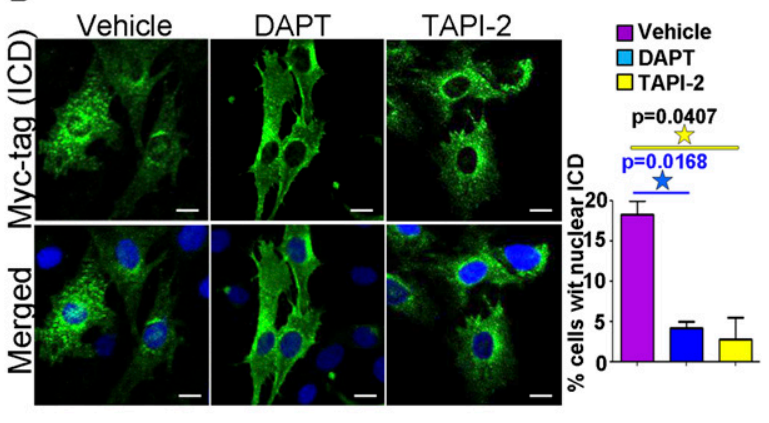

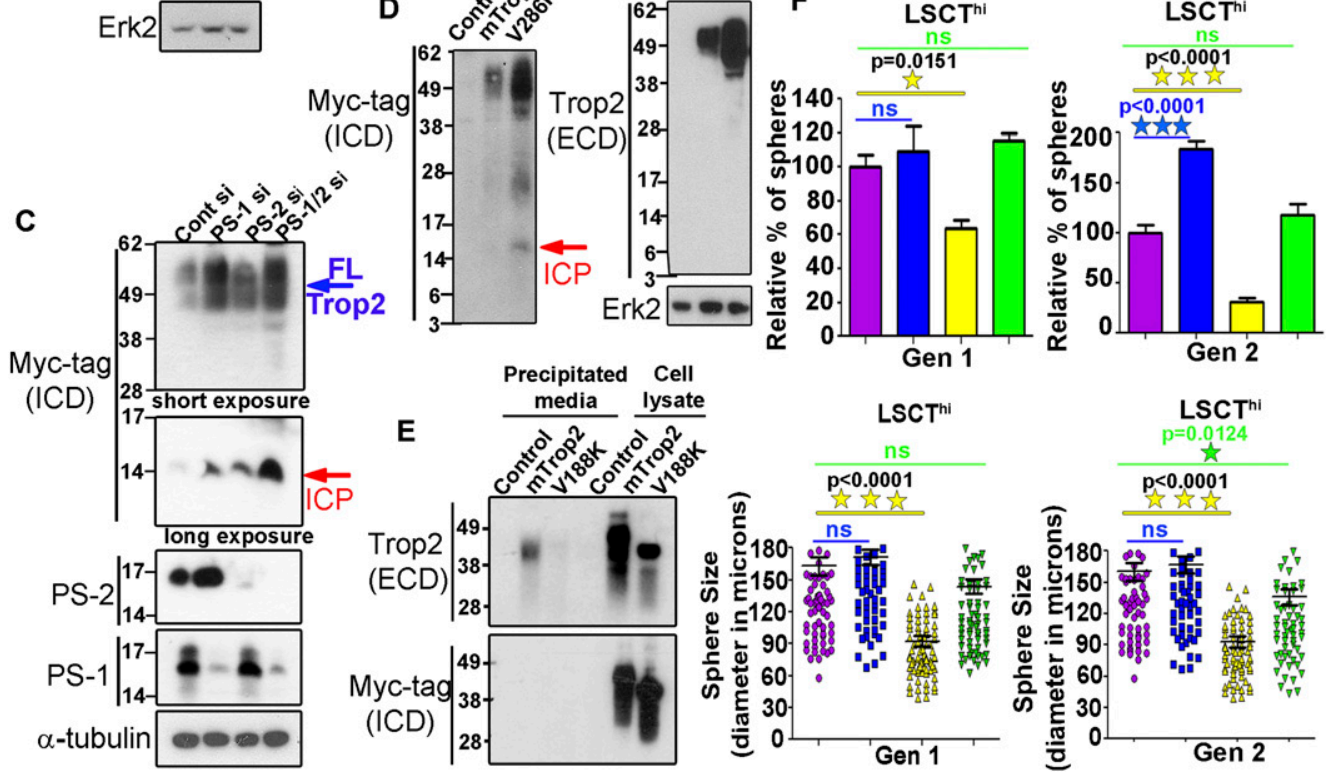

Figure 3. Trop2 is cleaved by RIP. Trop2 cleavage mutants are deficient in inducing self-renewal in vitro. $(A)$ PEB-Trop2-Myc tag cells were treated with either DMSO (vehicle), DAPT, or TAPI-2, followed by Western blot. The red arrow indicates accumulated ICP. The ICD cannot be detected without immunoprecipitation (IP). The blue arrow indicates full-length mTrop2 (FLTrop2). Fulllength Trop2 appears between 35 and $50 \mathrm{kD}$. One out of three independent experiments is shown. Schematic representation of the generation of $15-\mathrm{kD}$ ICP upon treatment with DAPT is shown on the right. (B) TRAMP-C2 expressing mTrop2 with a C-terminal Myc tag to follow the ICD were treated with DMSO (vehicle), DAPT, or TAPI-2. Twenty-four hours post-treatment, cells were fixed and stained with anti-Myc tag or anti-ECD antibody and DAPI and subjected to confocal microscopy. Bar, $10 \mu \mathrm{m}$. One out of two independent experiments is shown. The graph represents the percentage of cells with nuclear ICD. (C) PEB expressing Trop2Myc tag cells were transfected with nontargeting (Cont), PS-1, PS-2, or both PS-1 and PS-2 siRNA, followed by Western blot with the indicated antibodies. Similar to DAPT treatment, knockdown of presenilins caused ICP appearance, as indicated by the red arrow. The blue arrow shows full-length Trop2 (FLTrop2). One out of three independent experiments is shown. (D) PEB cells were infected with lentivirus expressing RFP (control), mTrop2-Myc tag and RFP (mTrop2), or V286K-Myc tag (V286) mutant. Cells were lysed and subjected to Western blot with antibodies against Myc tag or the ECD of Trop2. The red arrow shows ICP appearance in V286K, demonstrating not fully processed Trop2. One out of three independent experiments is shown. (E) Serumfree medium from TRAMP-C2 cells transduced with RFP lentivirus (control), mTrop2-Myc tag (mTrop2), or V188K-Myc tag (V188K) was precipitated, followed by Western blot using commercial anti-ECD antibody or anti-Myc tag recognizing the ICD. Only the ECD of Trop2 was detected in the precipitated media from cells expressing wild-type Trop2, but not from cells expressing V188K. Two independent experiments were performed. $(F)$ LSCT $^{\text {hi }}$ cells were isolated by FACS, and an equal number of cells was infected with RFP (control), mTrop2, V286K, or V188K mutants expressing lentivirus and plated in triplicate. Sphere number and diameter in microns were quantified after Gen 1 and Gen 2. (Left graphs) Sphere number in each sample is presented as the percentage normalized to the RFP-infected spheres (control). Data are represented as mean \pm SEM. Sphere size measured by sphere diameter in microns is presented in the right graphs. Purple bars represent RFP (control), blue bars indicate mTrop2, yellow bars represent V286K mutant, and green bars indicate V188K mutant-infected spheres. Each sample in the experiment was plated in triplicate. Three independent experiments were performed. 
distinct processes in defined cell populations even though both proteins are regulated by RIP.

\section{Trop2 is cleaved at two distinct sites}

While EpCAM cleavage sites still remain unknown, Notch1 processing sites have been well characterized (Schroeter et al. 1998; Mumm et al. 2000). Based on Notch cleavage sites (Schroeter et al. 1998; Mumm et al. 2000), we predicted the likely TACE cleavage site within the ECD (between Ala187 and Val188) and $\gamma$-secretase cleavage site within the transmembrane domain (between Gly285 and Val286). Each valine was substituted to a lysine (V188K and V286K). PEB cells were transduced with the $\gamma$-secretase cleavage mutant (V286K), TACE cleavage mutant (V188K), wild-type Trop2-Myc tag (mTrop2), or RFP (control) lentivirus. V286K and V188K localize on the cell surface similarly to the wild-type Trop2, as measured by FACS (Supplemental Fig. S6A). We tested whether mutation at the $\gamma$-secretase cleavage site would result in the appearance of ICP. Similar to treatment with DAPT, accumulation of ICP was observed in the V286K mutant but not in the wild-type Trop2 (Fig. $3 \mathrm{D})$. We further tested whether the V188K mutant is cleaved. Precipitated medium from prostate cells expressing TACE cleavage mutant (V188K), wild-type Trop2-Myc tag (mTrop2), or RFP (control) lentivirus was subjected to Western blot analysis with antibodies against Myc tag (ICD) and the ECD. Cleaved and shed ECD was detected in the medium from cells expressing wild-type Trop2 (mTrop2) but not from the cells expressing the V188K cleavage mutant (Fig. 3E).

\section{Cleavage of Trop2 is required for its self-renewal and proliferative activity}

To address whether Trop2 processing by RIP is necessary to carry out its self-renewal function, we tested the V286K and V188K mutants in the sphere assay (Fig. 3F; Supplemental Fig S6B). LSCT ${ }^{\text {hi }}$ cells were infected with lentivirus expressing RFP (control), mTrop2, V286K, or V188K cleavage mutants and plated in the in vitro sphere assay (Fig. 3F, Supplemental Fig S6B). While heightened levels of wild-type Trop2 promoted sphere formation, the cleavage mutants V286K and V188K failed to enhance self-renewal (Fig. 3F; Supplemental Fig S6B). Moreover, V286K negatively affected sphere formation (Fig. 3F; Supplemental Fig S6B). These results demonstrate that V286K may have a dominant-negative effect on endogenous Trop2, while V188K acts as a loss-of-function mutant. Pharmacological inhibition of Trop2 cleavage was also tested in the sphere assay. DAPT and TAPI-2 negatively impacted sphere formation in $\mathrm{LSCT}^{\text {hi }}$ cells by reducing sphere number and size (Supplemental Fig. S7A).

We asked whether Trop2 overexpression increases the percent of cycling cells in benign epithelial cells, as has been reported in cancer cells (Cubas et al. 2010). Both cleavage mutants $\mathrm{V} 286 \mathrm{~K}$ and $\mathrm{V} 188 \mathrm{~K}$ failed to increase proliferation and demonstrated cell cycle profiles similar to the control, while heightened Trop2 led to an increase in cycling cells (Supplemental Fig. S7B). Taken together, the combined genetic and small molecule inhibition results demonstrate that cleavage of Trop2 is necessary for its enhanced growth and self-renewal activity in Trop $2^{\text {hi }}$ cells.

\section{Trop2 is cleaved in human prostate cancer}

We next asked whether cleavage of Trop2 is observed in human prostate cancer. Frozen serial sections $(4-\mu \mathrm{m}$ thickness) of tissues from 11 prostate cancer patients containing both benign and cancer regions were subjected to coimmunofluorescence with antibodies against the ICD and ECD of human Trop2 or H\&E staining (Fig. 4; Supplemental Fig. S8). Stained tissues were analyzed for ICD and ECD cellular localization by confocal microscopy. Nuclear ICD was detected only in the cancer specimens but not in the benign counterparts in six out of the 11 patient samples analyzed (Fig. 4; Supplemental Fig. S8). The ECD was detected only in the cytoplasm and

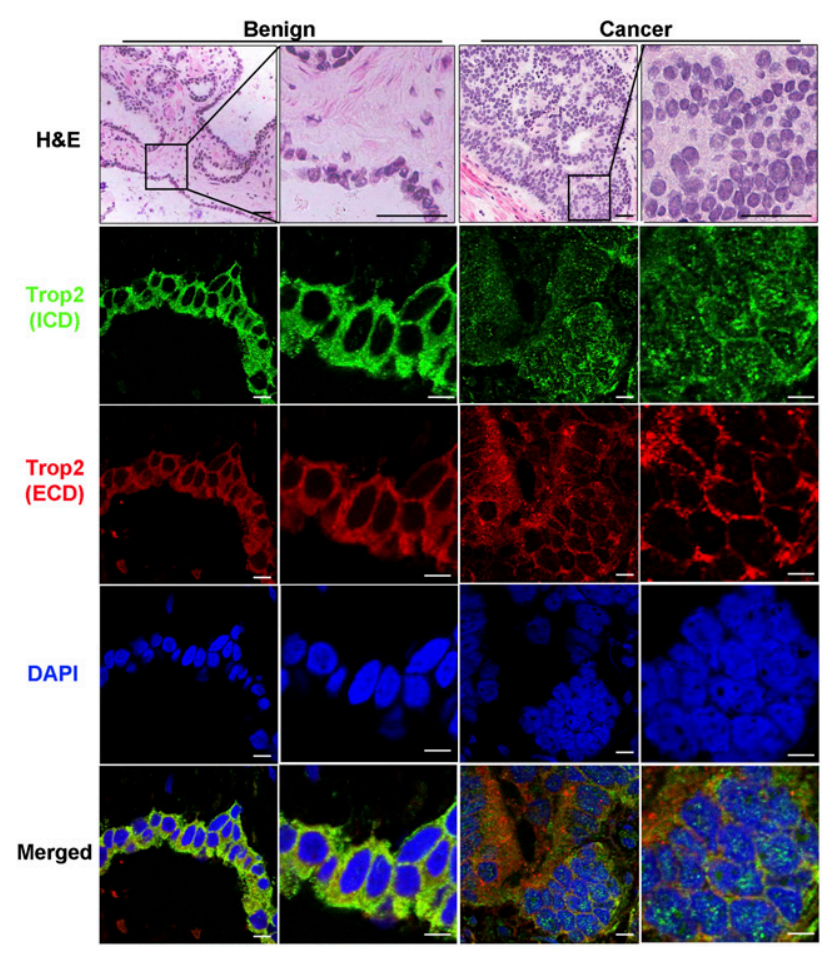

Figure 4. Nuclear localization of the ICD is found in human prostate cancer. Immunofluorescence with anti-ECD and antiICD antibodies and DAPI of tissues from human prostate cancer patients. One out of 11 patient samples is shown. A benign region is presented in the left two columns, and a cancer region is shown in the right two columns. Histology is shown in the top two panels by H\&E staining. Bars, $100 \mu \mathrm{m}$. ICD nuclear localization can be detected only in cancer but not in the benign prostate (green). The ECD is found in the cytoplasm and on the membrane in the benign and cancer regions (red). DAPI demonstrates nuclear staining (blue). Merged images are presented in the bottom panels. In the immunofluorescence images to visualize ICD and ECD localization, bars represent $10 \mu \mathrm{m}$. 
on the membrane in all patient samples in both cancer and benign regions (Fig. 4; Supplemental Fig. S8). These results indicate that cleavage and activation of Trop2 are associated with human prostate cancer.

\section{Heightened expression of the ICD initiates PIN in vivo}

To address the link between self-renewal and proliferative activity of Trop 2 and its role in prostate tumorigenesis, we tested whether heightened Trop2 is sufficient to transform naive epithelium and whether activation through cleavage is required for its function (Fig. 5A). We hypothesized that the expression of ICD alone will mimic active Trop2, while a cleavage mutant will represent a loss of function in vivo. Full-length mTrop2 was compared with $\gamma$-secretase cleavage mutant V286K, RFP alone, ICD to mimic active Trop2, and a potent oncogenic signal such as activated AKT in the in vivo regeneration assay (Fig. $5 B)$. Infection efficiency was assessed by the presence of the RFP or GFP color marker carried by each lentiviral vector. Enhanced expression of V286K, mICD, mTrop2, or AKT was confirmed by immunofluorescence or immunohistochemistry analysis in three independent grafts. Trop2 was able to drive hyperplasia, while the ICD was more potent, giving rise to lesions observed upon activation of AKT that morphologically resemble PIN, a precursor to carcinoma (Fig. 5B; Bostwick and Montironi 1995). V286K regenerated grafts resembled the RFP control and showed normal tubule regeneration, demonstrating that cleavage and activation of Trop2 is required for its transformation activity (Fig. 5B). The ECD alone was also sufficient to induce hyperplasia in the in vivo prostate regeneration assay (Supplemental Fig. S9).

\section{Trop2 signals through $\beta$-catenin}

Our findings that LSCT ${ }^{\text {hi }}$ cells express higher levels of $\beta$-catenin when compared with LSCT $^{\text {lo }}$ (Supplemental Fig. S2) led us to investigate whether Trop2 signals through $\beta$-catenin. A polyclonal antibody against the ICD was generated in our laboratory to assess whether the ICD and $\beta$-catenin physically interact. Due to higher levels of endogenous $\beta$-catenin in TRAMP-C2 cells, TRAMP-C2 cells expressing Trop2-Myc tag were used in immunoprecipitation experiments. The ICD coimmunoprecipitates with endogenous $\beta$-catenin (Fig. 6A). We next performed immunoprecipitation with an antibody against the ECD of Trop2. $\beta$-Catenin was not detected in the ECD immunoprecipitants (Fig. 6B). We set out to investigate whether Trop2 is involved in $\beta$-catenin signaling. Overexpression of Trop2 led to up-regulation of the $\beta$-catenin downstream targets cyclin D1 and c-myc, demonstrating that Trop2 signals through $\beta$-catenin (Fig. 6C,D; Supplemental Fig. S10).

\section{$\beta$-Catenin is required for Trop2-driven self-renewal and hyperplasia}

Having demonstrated that $\beta$-catenin binds to Trop 2 and is involved in Trop2 signaling, we next investigated localization of the ICD and $\beta$-catenin in human prostate tissues. Nuclear $\beta$-catenin was found in the cancer region of three out of 11 patient samples analyzed, all of which express nuclear Trop2 ICD (Fig. 7A; Supplemental Fig. 11). Importantly, $\beta$-catenin colocalized with Trop 2 ICD in all three patients (Fig. 7A; Supplemental Fig. 11). Nuclear colocalization was restricted to the cancer region and was not observed in the benign region (Fig. 7A; Supplemental Fig. 11).

To determine whether the biological activity of Trop2 is dependent on $\beta$-catenin, we investigated the link between $\beta$-catenin and Trop2-driven self-renewal and hyperplasia. Trop2 was overexpressed in wild-type or $\beta$-catenin-deleted epithelium and tested for self-renewal potential. LSCT ${ }^{\text {hi }}$ cells from $\beta$-catenin conditional knockout mice $(\operatorname{lox} \mathrm{P} / \mathrm{loxP})$ were infected with either mTrop2and RFP-expressing or RFP-expressing lentivirus and either Cre recombinase- and GFP-expressing (to drive excision of loxP sites, $\beta$-cat $\left.{ }^{-/-}\right)$or GFP-expressing $\left(\beta-\mathrm{cat}^{+/+}\right)$ lentivirus and plated in the sphere assay (Fig. 7B; Supplemental Fig. S12). Trop2 was able to drive an increase in sphere formation only in the presence of $\beta$-catenin (Fig. 7B; Supplemental Fig. S12). Loss of $\beta$-catenin abrogated the self-renewal driven by heightened Trop2 and showed baseline self-renewal activity comparable with the control.

To assess whether the activity of Trop2 is dependent on $\beta$-catenin in vivo, Trop2 was overexpressed in wild-type or $\beta$-catenin-deleted epithelium and tested for the ability to drive hyperplasia in the in vivo regeneration assay. Dissociated primary prostate cells from $\beta$-catenin conditional knockout mice were transduced with mTrop2 and RFP and either Cre recombinase-and GFP-expressing $\left(\beta\right.$-cat $\left.{ }^{-/-}\right)$or GFP-expressing $\left(\beta-\mathrm{cat}^{+/+}\right)$lentivirus. Transduced prostate cells were combined with UGSM cells and subjected to in vivo regeneration. Upon histological analysis of the recovered grafts, we observed that Trop2 was able to induce hyperplasia only in the presence of $\beta$-catenin (mTrop $2 / \beta$-cat ${ }^{+/+}$) (Fig. 7C). Loss of $\beta$-catenin blocked the hyperplasia induced by heightened Trop2 (mTrop2/ $\beta$ - $\mathrm{cat}^{-/-}$) and showed normal prostate tubule regeneration (Fig. $7 \mathrm{C}$ ). These findings demonstrate that Trop2 function requires the presence of $\beta$-catenin (Fig. 7B,C).

\section{Discussion}

Recent evidence suggests that many human cancers arise from transformed stem cells due to their enhanced selfrenewal capacity (Visvader, 2011). Identifying the factors that regulate self-renewal will enhance our understanding of the tumorigenic process and lead to the design of novel cancer therapies. We showed here that Trop2 is a new regulator of stem/progenitor activity in the prostate. Trop2 controls self-renewal, proliferation, and tissue hyperplasia through two cleavage products (ICD and ECD) generated by RIP. We speculate that elevated expression of Trop2 is selected for in cancer due to its capacity to enhance self-renewal and proliferation.

We found that Trop2 cleavage is mediated by both PS-1 and PS-2 in the $\gamma$-secretase complex. Our observations that knockdown of PS-1 and, to lesser extent, PS-2 

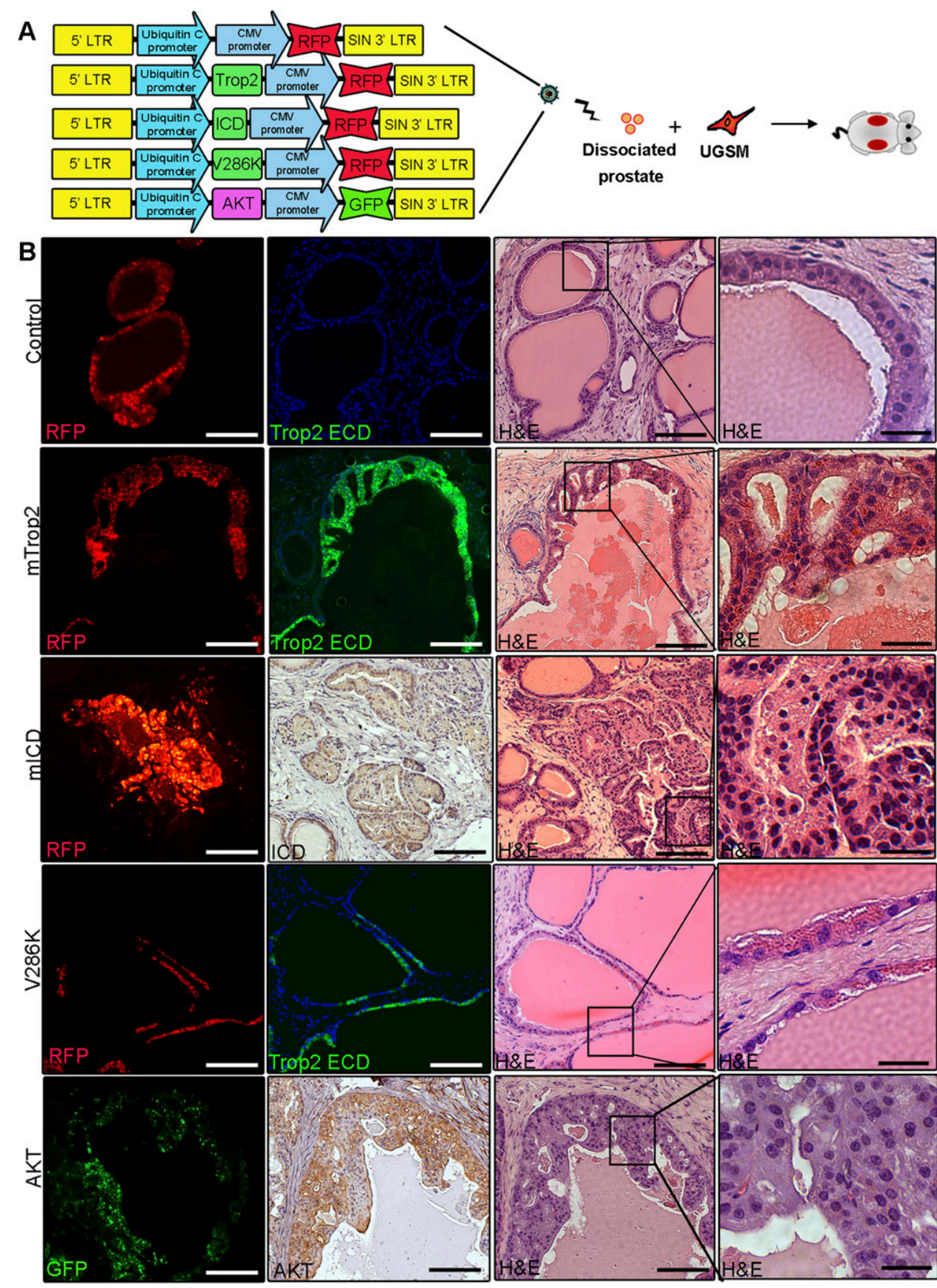

Figure 5. Trop2 and the ICD alone drive hyperplasia in vivo. (A) Schematic representation of the in vivo regeneration assay and lentiviral construct used. (B) Dissociated primary prostate cells were transduced with lentivirus expressing RFP (control), Trop2, mICD, V286K, or AKT. Infected prostate cells were combined with UGSM and subjected to the in vivo regeneration assay. Eight weeks later, grafts were recovered, fixed in formalin, and sectioned for histological analysis. The left panels represent immunofluorescence (IF) showing infected tubules, and the middle panel shows immunofluorescence with anti-Trop2 ECD or immunohistochemistry (IHC) with anti-ICD or anti-AKT, respectively. The right two panels show the histology of the recovered grafts. One out of three independent experiments is shown. Bars: right panels, 200 or $50 \mu \mathrm{m}$.

resulted in elevated surface Trop2 suggest that PS-1 is the predominant enzyme involved in Trop2 processing. In contrast, EpCAM cleavage by RIP is carried out strictly by PS-2 (Maetzel et al. 2009).

Analysis of Trop2 and EpCAM localization in the mouse prostate revealed that these related proteins have different expression patterns, which may have a biological consequence. While we demonstrated that Trop2 regulates self-renewal and tissue regeneration, no evidence has been provided to support a functional role for EpCAM in adult stem/progenitor cells. The Notch family of receptors provides one example of highly homologous 
A

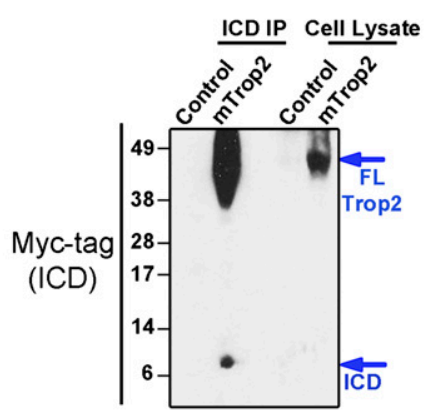

B



C

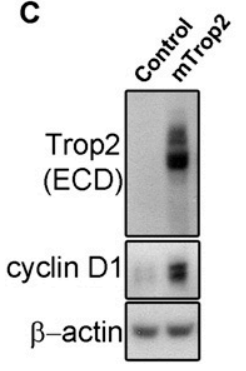

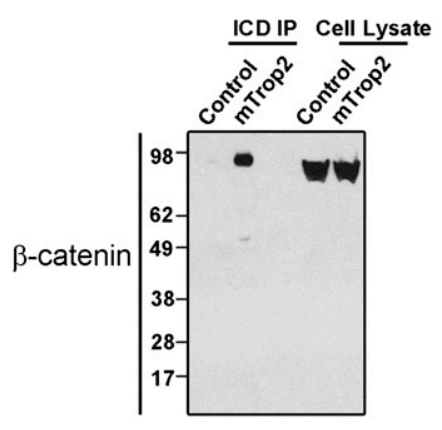

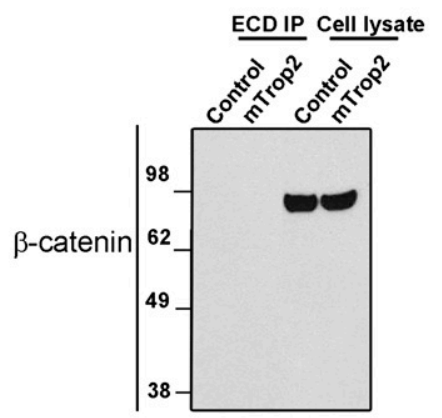

D

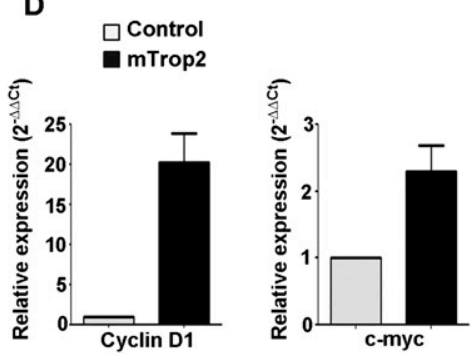

Figure 6. Trop 2 signals through $\beta$-catenin. $(A)$ Trop2 ICD binds $\beta$-catenin. Cell lysates from TRAMP-C2 cells expressing either RFP (control) or the mTrop2-Myc tag and RFP (mTrop2) were subjected to immunoprecipitation using antibodies against the ICD raised in our laboratory (ICD immunoprecipitation), followed by Western blot with anti-Myc tag (ICD) (on the left) or anti- $\beta$-catenin (on the right) antibodies. Trop2 is shown between 35 and $50 \mathrm{kD}$ due to high glycosylation. One out of three independent experiments is shown. $(B)$ Lysates from TRAMP-C2 cells transduced with a RFPexpressing lentivirus (control) or mouse Trop2 and RFP lentivirus (mTrop2) were subjected to immunoprecipitation with polyclonal antibody against the ECD of Trop2, followed by Western blot with anti-Myc tag (ICD) or anti- $\beta$-catenin antibodies. No interaction between the ECD and $\beta$-catenin was observed. One out of three independent experiments is presented. $(C)$ Overexpression of Trop2 increases $\beta$-catenin target genes. Lysates from PEB expressing RFP (control) or mouse Trop2 (Trop2) were subjected to SDS-PAGE followed by Western blot with anti-Trop2 ECD, anti-cyclin D1, antic-myc, or anti- $\beta$-actin antibodies. One out of three independent experiments is shown. (D) RNA isolated from PEB expressing RFP (control) or mouse Trop2 (Trop2) was subjected to cDNA synthesis, and quantitative PCR was performed in triplicate using mouse-specific cyclin D1 and c-myc primers. One out of three independent experiments is shown. Fold change of cyclin D1 and c-myc mRNA levels is normalized to GAPDH (internal control). proteins carrying differential functions. Notch1, Notch2, and Notch3 are expressed in distinct cell populations within the thymus, are regulated by RIP, and induce analogous signaling cascades (Felli et al. 1999). Both Notch1 and Notch2 are expressed in myeloid progenitor cells, but they are each cleaved in response to different cytokines, supporting unique downstream signaling patterns (Bigas et al. 1998).

Upon activation by RIP, we determined that Trop2 signals through $\beta$-catenin. Trop2 was not able to induce self-renewal and hyperplasia upon $\beta$-catenin loss, demonstrating that the Trop2 self-renewal and transformation function is dependent on $\beta$-catenin activity. Abnormal $\beta$-catenin localization is found in $71 \%$ of prostate cancer patients, and high levels of $\beta$-catenin are associated with metastatic prostate cancer (Yardy and Brewster 2005; Yardy et al. 2009). Elevated levels of Trop2 are found in localized and metastatic human prostate cancer compared with benign prostate tissue ( $\mathrm{T}$ Stoyanova, AS Goldstein., J Huang, H Zhang, and ON Witte, unpubl.). We now demonstrate that nuclear $\beta$-catenin colocalizes with the ICD within the nucleus. This colocalization is restricted to cancer regions and does not occur in the benign tissues. Nuclear ICD is also observed in prostate cancer patients with low levels of nuclear $\beta$-catenin. Additional analysis will need to be performed to investigate the relationship between high nuclear $\beta$-catenin that colocalizes with the ICD and the clinical outcome of prostate cancer patients.

Due to its elevated expression in a broad range of epithelial cancers, Trop2 is a promising target for therapeutic intervention. Antibodies against Trop2 were used as carriers of radioactive elements or toxins to the tumor sites and demonstrated great anti-cancer efficacies in xenograft models of multiple cancers (Shih et al. 1995; Stein et al. 1997; Govindan et al. 2004; Cardillo et al. 2011). Trop 2 antibodies can also be used as direct therapeutic effectors. Nonconjugated Trop 2 antibodies exhibit potent anti-cancer activity in cancer xenografts of many epithelial cancers (Young et al. 2008; Alberti 2012). These studies suggest that the antibodies interfere with Trop2 signaling, most likely by compromising cancer self-renewal and proliferation. Thorough interrogation of the functional role of Trop2 in stem/progenitor self-renewal and transformation and of the molecular mechanisms of Trop2 function defined in our study will be applicable in developing novel targeted therapies. Activation and signaling driven by Trop2 require RIP, as cleavage mutants 

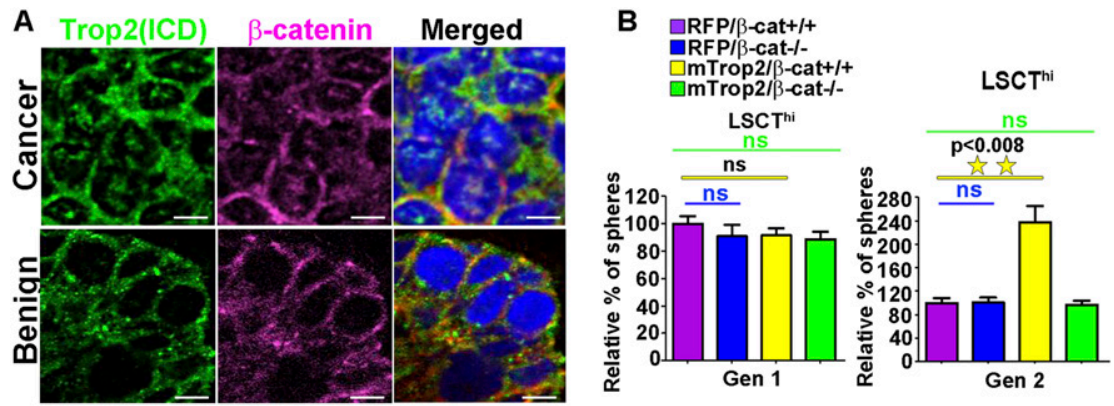

C


Trop2/DAPI

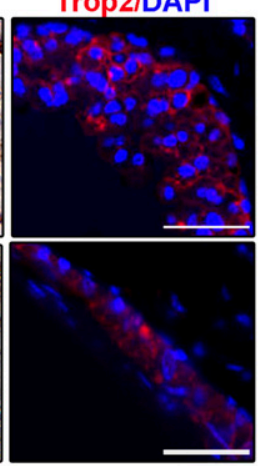

D
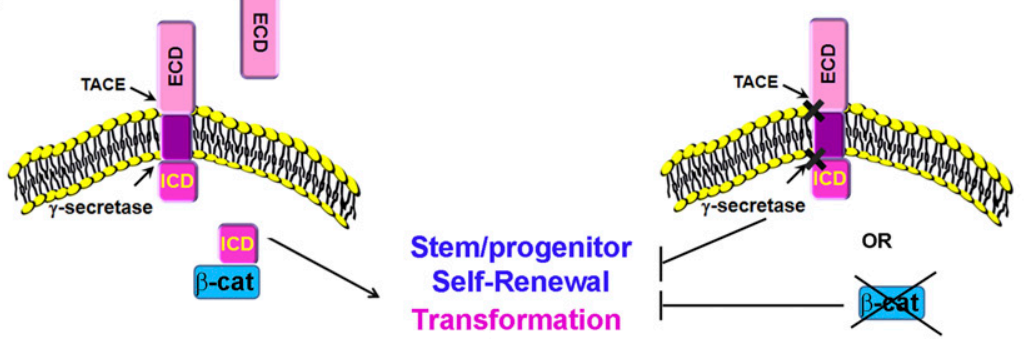

Figure 7. Trop2 regulates self-renewal and transformation though $\beta$-catenin. (A) Immunofluorescence of tissues from human prostate cancer patients with anti-ICD and anti$\beta$-catenin antibodies and DAPI. One out of 11 patient samples is shown. A cancer region is presented in the top panels, and a benign region is shown in the bottom panels. The ICD (green) colocalizes with $\beta$-catenin (pink) in the nucleus only in cancer but not in the benign prostate. DAPI demonstrates nuclear staining (blue). Merged images are presented in the bottom panels. Bars, $10 \mu \mathrm{m}$. (B) Trop2-mediated enhanced self-renewal requires $\beta$-catenin. Isolated progenitor cells (LSCT ${ }^{\text {hi }}$ ) from $\beta$-catenin conditional knockout mice $(\operatorname{lox} \mathrm{P} / \operatorname{lox} \mathrm{P})$ were infected with either mTrop2- and RFPexpressing lentivirus or control RFP (RFP) and either Cre recombinase- and GFPexpressing lentivirus (to drive excision of loxP sites, $\beta$-cat ${ }^{-/-}$) or control GFP-expressing lentivirus $\left(\beta-\right.$ cat $\left.^{+/+}\right)$. Infected cells were plated in triplicate in the sphere-forming assay, and sphere numbers were quantified $7 \mathrm{~d}$ post-plating for Gen 1 and further passaged to Gen 2. (Left graphs) Sphere number in each sample is presented as the percentage normalized to the RFP control. Data are represented as mean \pm SEM. One out of three independent experiments is shown. $(C)$ Dissociated primary prostate cells from $\beta$-catenin conditional knockout mice were transduced with either mTrop2and RFP-expressing lentivirus and either Cre recombinase (mTrop $2 / \beta-\mathrm{cat}^{-/-}$) or GFP $\left(\mathrm{mTrop} 2 / \beta-\mathrm{cat}^{+/+}\right)$and combined with UGSM and subjected to the in vivo regeneration
ogical analysis. The left four panels represent assay. Eight weeks later, grafts were recovered, fixed in formalin, and sectioned for histological analysis. The left four panels represent Trop2 ECD and DAPI. Bars: right panels, 100 or $50 \mu \mathrm{m}$. (D) Trop2 is activated through RIP by the TACE and $\gamma$-secretase complex. RIP of Trop2 results in shedding of the ECD and release of the ICD to the nucleus. The ICD promotes self-renewal through $\beta$-catenin. The models suggest that the ICD promotes transformation through its self-renewal activity.

were deficient in self-renewal and transformation, suggesting that inhibition of regulated processing might inhibit proliferation and survival in prostate cancer cells (Fig. 7D). Small molecules able to block Trop2 proteolysis or monoclonal antibodies that interfere with Trop2 processing and activation could represent a promising strategy to target not only prostate, but other epithelial cancers exhibiting elevated levels of Trop2 (Fig. 7D).

\section{Materials and methods}

\section{Vector production}

Mouse Trop2 was PCR-amplified from pCR-BluntII-TOPO vector containing the ORF of mouse Trop2 (purchased from Open Biosystems). Trop2 was modified by adding a Myc tag at the $\mathrm{C}$-terminal end, and $\mathrm{XbaI}$ restriction digest sites were added to both ends. The amplified Trop2 was subcloned into the XbaI site of FUCRW downstream from the ubiquitin promoter. Trop2 ICD was PCR-amplified, and XbaI and EcoR1 sites were added, followed by subcloning into FUCRW downstream from the ubiquitin promoter.

To generate V188K and V286K Trop2 mutants, mouse Trop2 was subcloned in pBSKSII vector. Stratagene's QuikChange sitedirected mutagenesis kit was used to introduce a single-aminoacid change with the following oligonucleotides: V188K (CTT CCTATCCGCGAAACACTATGAGGAGCCCACC and GGTG GGCTCCTCATAGTGTTTCGCGGATAGGAAG) and V286K (TAGCGGTAGTGGCTGGTAAGGTGGTCTTGGTGGT and ACCACCAAGACCACCTTACCAGCCACTACCGCTA). V188K and V286K Trop2 mutants were subcloned into the FUCRW XbaI and EcoR1 sites downstream from the ubiquitin promoter.

The BLOCK-iT PolII miRNAi expression vector kit was used for generation of miRNA scrambled-GFP and miRNA Trop2-GFP with the following oligonucleotides: miRNA scrambled-GFP (TGCTGAAATGTACTGCGCGTGGAGACGTTTTGGCCACT GACTGACGTCTCCACGCAGTACATTTCAGG) and miRNA Trop2-GFP (TGCTGAACTCAATGAGGATGTGGTGGGTTTT GGCCACTGACTGACCCACCACACTCATTGAGTTCAGG). 
Viral packaging

Third-generation lentiviral vectors FUCGW and FUCRW, derived from FUGW (Lois et al. 2002; Xin et al. 2006), were used for the construction of the mouse Trop2, ICD, ECD-Fc, V286K, and V188K cleavage mutant overexpression.

\section{Mouse strains}

C57BL/6 and CB1 $7^{\text {Scid/Scid }}$ mouse strains were purchased from The Jackson Laboratory. Mice were housed in the University of California at Los Angeles (UCLA) animal facilities under the regulation of the Division of Laboratory Animal Medicine.

\section{Prostate regeneration assays}

Housing, maintenance, and all surgical procedures were undertaken in compliance with the regulations of the Division of Laboratory Animal Medicine of UCLA. All experimental procedures were approved by the Division of Laboratory Animal Medicine of UCLA. The details of the regeneration process have been explained previously (Lukacs et al. 2010).

\section{Prostate cell dissociation, cell sorting, and in vitro sphere assay}

Isolation of prostate epithelial cells, antibody staining, and FACS for $\mathrm{LSCT}^{\mathrm{lo}}$, LSCThi, and luminal cells were performed as previously described (Goldstein et al. 2008; Lukacs et al. 2010). Five thousand to 50,000 sorted LSCT ${ }^{\text {hi }}$ or LSCT ${ }^{\text {lo }}$ prostate cells were used for sphere assay following the previously published protocol (Lukacs et al. 2010).

\section{Prostate patient samples}

Patient samples were obtained from the UCLA Translational Pathology Core Laboratory (TPCL), which is authorized by the UCLA Institutional Review Board (IRB) to distribute annonymized tissue to researchers. All tissues were from prostatectomy specimens that contained prostate cancer as well as benign prostate. Fresh prostates were serially sectioned into five to seven slices, depending on their sizes. Levels 2 and 4 were submitted to the TPCL. Each level was divided into four quadrants, and a rapidfrozen section diagnosis was performed. Cancer and benign areas were clearly marked on the frozen section slides, and the frozen tissue was stored in $-80^{\circ} \mathrm{C}$ freeze. Additional sections were cut from the tissue for $\mathrm{H} \& \mathrm{E}$ staining and immunofluorescence studies.

\section{Western blot and immunoprecipitation}

For Western blot assay, cells were washed twice with $1 \times \mathrm{PBS}$ and lysed in RIPA buffer contacting $50 \mathrm{mM}$ Tris- $\mathrm{HCl}(\mathrm{pH} 8.0), 150$ $\mathrm{mM} \mathrm{NaCl}, 0.1 \%$ SDS, $0.5 \%$ SD, $1 \%$ NP-40, 1 mM EDTA, cocktail protease inhibitors (Roche), and phosphatase inhibitor 1 and 2 (Sigma). Extracts $(10-50 \mu \mathrm{g})$ were subjected to sodium dodecyl sulfate- $10 \%$ polyacrylamide gel electrophoresis, followed by blotting to nitrocellulose with the indicated antibodies. For immunoprecipitation, cells were lysed by suspension in $2 \mathrm{vol}$ of buffer containing $0.4 \mathrm{M} \mathrm{NaCl}, 20 \mathrm{mM}$ Tris- $\mathrm{HCl}$ (pH 7.5), $0.1 \%$ NP-40, 5\% (v/v) glycerol and cocktail protease inhibitors (Roche), and phosphatase inhibitor 1 and 2 (Sigma). Extracts $(500 \mu \mathrm{g})$ were subjected to immunoprecipitation with $1 \mu \mathrm{g}$ of anti-ICD, anti-ECD or with isotype-matched immunoglobulin (IgG) followed by Western blot.

\section{Immunofluorescence}

For cytospins and immunofluorescence, $1 \times 10^{5}$ cells were spun on slides and fixed in ice-cold acetone, followed by staining with the indicated antibodies. Antibodies and dilutions are provided in the Supplemental Material. Slides were counterstained with DAPI and visualized with confocal fluorescence microscopy.

\section{Immunohistochemistry}

Indicated tissues were fixed in $10 \%$ buffer formalin and paraffinembedded. Four-micron sections were deparaffinized in xylene and rehydrated in 100\%, 95\%, and $70 \%$ ethanol. Antigen retrieval was performed with citrate buffer ( $\mathrm{pH}$ 6.0) for $20 \mathrm{~min}$ at $95^{\circ} \mathrm{C}$. Sections were further blocked using mouse-on-mouse blocking reagents (Vector Laboratories, BMK-2202). The sections were incubated with the indicated antibodies overnight. Slides were washed with $1 \times$ PBS and incubated with anti-mouse HRP or anti-rabbit HRP antibodies (DAKO) for $1 \mathrm{~h}$, developed with HRP substrate (DAKO), and counterstained with hematoxylin.

\section{Antibodies}

Western blot analysis and immunoprecipitation experiments were performed using the following antibodies: anti-Trop2 ECD (R\&D Systems, FAB1122A, FAB1122F, and BAF1122), anti-Myc tag (Cell Signaling, no. 2276S and no. 2278), anti Erk-2 (Santa Cruz Biotechnology, sc-154), anti- $\alpha$-tubulin (Santa Cruz Biotechnology, sc-5286), anti-PS-1 (Cell Signaling, no. 5643S), anti-PS-2 (Cell Signaling, no. 2192), anti-cyclinD1 (Cell Signaling, no. 2926), anti- $\beta$-catenin (Transduction Laboratories, 610454), anti-EpCAM (Abcam, ab68892), and anti-EpCAM C terminus (Epitomics, 1144-1).

$\operatorname{siRNA}$

On-Target Plus smart pool siRNA targeting mouse PS-1 and PS-2 and nontargeting siRNA control were purchased from Dharmacon as previously described (Zhao et al. 2010): PS-1 siRNA (CC AAAGGCCCACUUCGUAU, GAAGGAUAGCUCCGAGUAA, GGAGCAUUCUAACGAGUGA, and CCGGAAGGACGGUC AGCUA) and PS-2 siRNA (CCUCAAGUAUGGGGCGAAA, CUAUCAAGUCUGUGCGUUU, AAGAGGACCCGGACCGC UA, and CAGCUUGCCUGUCGGAGCA).

\section{Inhibitors}

DAPT $(10 \mu \mathrm{M})$ (Sigma Aldrich, D5942) or $40 \mu \mathrm{M}$ TAPI-2 (Enzo Life Sciences, BML-PI135-0001) were used.

\section{Statistical analysis}

All experiments were performed with at least three different primary cultures or animals in independent experiments. Significance was evaluated by Student's $t$-test. Data are presented as mean \pm SEM.

\section{ICD antibody generation}

Anti-ICD rabbit polyclonal antibody was generated by Open Biosystems, following the Custom Polyclonal Antibody Production protocol. Briefly, KLH-conjugated mouse and human ICD peptides were used as antigens (mouse, TKRRKSGKYKK VELKELGEMRSEPSL; human, TNRRKSGKYKKVEIKELGELR KEPSL). Immunoglobulin fraction was purified from the rabbit serum through protein $\mathrm{A} / \mathrm{G}$ purification. Purified immunoglobulins 
were tested by ELISA, immunoprecipitation, immunofluorescence, and immunohistochemistry for reactivity with the mouse and human ICD.

\section{Acknowledgments}

We thank Donghui Cheng for FACS, Mireille Riedinger for antiICD antibody generation, and Hong Zhang for human tissue preparation. We thank members of the Witte laboratory and Marta Morey for helpful comments and discussion on the manuscript, and Sandra Orellana for technical help. T.S. is supported by the California Institute for Regenerative Medicine Training Grant (TG2-01169) and the Department of Defense Prostate Cancer Research Program (PC110638). A.S.G. is supported by a Career Development Award from the SPORE in prostate cancer (Principal Investigator [PI]: Robert Reiter) and the Joe and Ali Torre-Prostate Cancer Foundation Young Investigator Award. J.M.D. is supported by the Department of Defense Prostate Cancer Research Program (PC101928) and UCLA Tumor Biology Program (Ruth L. Kirschstein Institutional National Research Service Award no. T32 CA009056). J.H. is supported by UCLA SPORE in Prostate Cancer (PI: Robert Reiter), Department of Defense Prostate Cancer Research Program grants W81XWH11-1-0227 (PI: Jiaoti Huang) and W81XWH-12-1-0206 (PI: Lily $\mathrm{Wu}$ ), and the National Cancer Institute (1R01CA158627-01; PI: Leonard Marks). J.H., A.S.G., and O.N.W. are supported by a Prostate Cancer Foundation Challenge Award (PI: Owen Witte). A.S.G. and O.N.W. are supported by a Prostate Cancer Foundation Creativity Award (PI: Owen Witte). O.N.W. is an Investigator of the Howard Hughes Medical Institute.

\section{References}

Alberti S. 2012. Anti-trop-2 monoclonal antibodies and uses thereof in the treatment and diagnosis of tumors. U.S. Patent US 20120052076.

Basu A, Goldenberg DM, Stein R. 1995. The epithelial/carcinoma antigen EGP-1, recognized by monoclonal antibody RS7-3G11, is phosphorylated on serine 303. Int I Cancer 62: 472-479.

Bigas A, Martin DI, Milner LA. 1998. Notch1 and Notch2 inhibit myeloid differentiation in response to different cytokines. Mol Cell Biol 18: 2324-2333.

Bostwick DG, Montironi R. 1995. Prostatic intraepithelial neoplasia and the origins of prostatic carcinoma. Pathol Res Pract 191: 828-832.

Brown MS, Ye J, Rawson RB, Goldstein JL. 2000. Regulated intramembrane proteolysis: A control mechanism conserved from bacteria to humans. Cell 100: 391-398.

Cardillo TM, Govindan SV, Sharkey RM, Trisal P, Goldenberg DM. 2011. Humanized anti-Trop-2 IgG-SN-38 conjugate for effective treatment of diverse epithelial cancers: Preclinical studies in human cancer xenograft models and monkeys. Clin Cancer Res 17: 3157-3169.

Chen G, Shukeir N, Potti A, Sircar K, Aprikian A, Goltzman D, Rabbani SA. 2004. Up-regulation of Wnt-1 and $\beta$-catenin production in patients with advanced metastatic prostate carcinoma: Potential pathogenetic and prognostic implications. Cancer 101: 1345-1356.

Cubas R, Zhang S, Li M, Chen C, Yao Q. 2010. Trop2 expression contributes to tumor pathogenesis by activating the ERK MAPK pathway. Mol Cancer 9: 253. doi: 10.1186/1476-4598-9-253.

Cunha GR, Lung B. 1978. The possible influence of temporal factors in androgenic responsiveness of urogenital tissue recombinants from wild-type and androgen-insensitive (Tfm) mice. J Exp Zool 205: 181-193.
De Strooper B, Annaert W, Cupers P, Saftig P, Craessaerts K, Mumm JS, Schroeter EH, Schrijvers V, Wolfe MS, Ray WJ, et al. 1999. A presenilin-1-dependent $\gamma$-secretase-like protease mediates release of Notch intracellular domain. Nature 398: $518-522$.

Dontu G, Abdallah WM, Foley JM, Jackson KW, Clarke MF, Kawamura MJ, Wicha MS. 2003a. In vitro propagation and transcriptional profiling of human mammary stem/progenitor cells. Genes \& Dev 17: 1253-1270.

Dontu G, Al-Haij M, Abdallah WM, Clarke MF, Wicha MS. 2003b. Stem cells in normal breast development and breast cancer. Cell Prolif 36: 59-72.

Dontu G, Jackson KW, McNicholas E, Kawamura MJ, Abdallah WM, Wicha MS. 2004. Role of Notch signaling in cell-fate determination of human mammary stem/progenitor cells. Breast Cancer Res 6: R605-R615. doi: 10.1186/bcr920.

Fang YJ, Lu ZH, Wang GQ, Pan ZZ, Zhou ZW, Yun JP, Zhang MF, Wan DS. 2009. Elevated expressions of MMP7, TROP2, and survivin are associated with survival, disease recurrence, and liver metastasis of colon cancer. Int J Colorectal Dis 24: 875-884.

Felli MP, Maroder M, Mitsiadis TA, Campese AF, Bellavia D, Vacca A, Mann RS, Frati L, Lendahl U, Gulino A, et al. 1999. Expression pattern of notch1, 2 and 3 and Jagged 1 and 2 in lymphoid and stromal thymus components: Distinct ligandreceptor interactions in intrathymic $\mathrm{T}$ cell development. Int Immunol 11: 1017-1025.

Fong D, Moser P, Krammel C, Gostner JM, Margreiter R, Mitterer M, Gastl G, Spizzo G. 2008a. High expression of TROP2 correlates with poor prognosis in pancreatic cancer. Br J Cancer 99: 1290-1295.

Fong D, Spizzo G, Gostner JM, Gastl G, Moser P, Krammel C, Gerhard S, Rasse M, Laimer K. 2008b. TROP2: A novel prognostic marker in squamous cell carcinoma of the oral cavity. Mod Pathol 21: 186-191.

Foster BA, Gingrich JR, Kwon ED, Madias C, Greenberg NM. 1997. Characterization of prostatic epithelial cell lines derived from transgenic adenocarcinoma of the mouse prostate (TRAMP) model. Cancer Res 57: 3325-3330.

Goldstein AS, Lawson DA, Cheng D, Sun W, Garraway IP, Witte ON. 2008. Trop2 identifies a subpopulation of murine and human prostate basal cells with stem cell characteristics. Proc Natl Acad Sci 105: 20882-20887.

Goldstein AS, Huang J, Guo C, Garraway IP, Witte ON. 2010. Identification of a cell of origin for human prostate cancer. Science 329: 568-571.

Govindan SV, Stein R, Qu Z, Chen S, Andrews P, Ma H, Hansen HJ, Griffiths GL, Horak ID, Goldenberg DM. 2004. Preclinical therapy of breast cancer with a radioiodinated humanized anti-EGP-1 monoclonal antibody: Advantage of a residualizing iodine radiolabel. Breast Cancer Res Treat 84: $173-182$.

Govindan S, Qu Z, Hansen H, Goldenberg D. 2009. RS7 antibodies. U.S. Patent US 7517964 B2.

Kobel M, Kalloger SE, Boyd N, McKinney S, Mehl E, Palmer C, Leung S, Bowen NJ, Ionescu DN, Rajput A, et al. 2008. Ovarian carcinoma subtypes are different diseases: Implications for biomarker studies. PLoS Med 5: e232. doi: 10.1371/ journal.pmed.0050232.

Lal M, Caplan M. 2011. Regulated intramembrane proteolysis: Signaling pathways and biological functions. Physiology 26: 34-44.

Lawson DA, Xin L, Lukacs RU, Cheng D, Witte ON. 2007. Isolation and functional characterization of murine prostate stem cells. Proc Natl Acad Sci 104: 181-186. 
Lobry C, Oh P, Aifantis I. 2011. Oncogenic and tumor suppressor functions of Notch in cancer: It's NOTCH what you think. I Exp Med 208: 1931-1935.

Lois C, Hong EJ, Pease S, Brown EJ, Baltimore D. 2002. Germline transmission and tissue-specific expression of transgenes delivered by lentiviral vectors. Science 295: 868-872.

Lukacs RU, Goldstein AS, Lawson DA, Cheng D, Witte ON. 2010. Isolation, cultivation and characterization of adult murine prostate stem cells. Nat Protoc 5: 702-713.

Maetzel D, Denzel S, Mack B, Canis M, Went P, Benk M, Kieu C, Papior P, Baeuerle PA, Munz M, et al. 2009. Nuclear signalling by tumour-associated antigen EpCAM. Nat Cell Biol 11: 162-171.

Maretzky T, Reiss K, Ludwig A, Buchholz J, Scholz F, Proksch E, de Strooper B, Hartmann D, Saftig P. 2005. ADAM10 mediates E-cadherin shedding and regulates epithelial cellcell adhesion, migration, and $\beta$-catenin translocation. Proc Natl Acad Sci 102: 9182-9187.

Memarzadeh S, Zong Y, Janzen DM, Goldstein AS, Cheng D, Kurita T, Schafenacker AM, Huang J, Witte ON. 2010. Cellautonomous activation of the PI3-kinase pathway initiates endometrial cancer from adult uterine epithelium. Proc Natl Acad Sci 107: 17298-17303.

Muhlmann G, Spizzo G, Gostner J, Zitt M, Maier H, Moser P, Gastl G, Zitt M, Muller HM, Margreiter R, et al. 2009. TROP2 expression as prognostic marker for gastric carcinoma. J Clin Pathol 62: 152-158.

Mulholland DJ, Xin L, Morim A, Lawson D, Witte O, Wu H. 2009. Lin $^{-} \mathrm{Sca}-1^{+} \mathrm{CD} 49 \mathrm{f}^{\text {high }}$ stem/progenitors are tumor-initiating cells in the Pten-null prostate cancer model. Cancer Res 69: 8555-8562.

Mumm JS, Schroeter EH, Saxena MT, Griesemer A, Tian X, Pan DJ, Ray WJ, Kopan R. 2000. A ligand-induced extracellular cleavage regulates $\gamma$-secretase-like proteolytic activation of Notch1. Mol Cell 5: 197-206.

Nakashima K, Shimada H, Ochiai T, Kuboshima M, Kuroiwa N, Okazumi S, Matsubara H, Nomura F, Takiguchi M, Hiwasa T. 2004. Serological identification of TROP2 by recombinant cDNA expression cloning using sera of patients with esophageal squamous cell carcinoma. Int I Cancer 112: 1029-1035.

Ohmachi T, Tanaka F, Mimori K, Inoue H, Yanaga K, Mori M. 2006. Clinical significance of TROP2 expression in colorectal cancer. Clin Cancer Res 12: 3057-3063.

Okabe M, Tsukahara Y, Tanaka M, Suzuki K, Saito S, Kamiya Y, Tsujimura T, Nakamura K, Miyajima A. 2009. Potential hepatic stem cells reside in $\mathrm{EpCAM}^{+}$cells of normal and injured mouse liver. Development 136: 1951-1960.

Ralhan R, Cao J, Lim T, Macmillan C, Freeman JL, Walfish PG. 2010a. EpCAM nuclear localization identifies aggressive thyroid cancer and is a marker for poor prognosis. $B M C$ Cancer 10: 331. doi: 10.1186/1471-2407-10-331.

Ralhan R, He HC, So AK, Tripathi SC, Kumar M, Hasan MR, Kaur J, Kashat L, MacMillan C, Chauhan SS, et al. 2010b. Nuclear and cytoplasmic accumulation of Ep-ICD is frequently detected in human epithelial cancers. PLOS ONE 5: e14130. doi: 10.1371/journal.pone.0014130.

Ranganathan P, Weaver KL, Capobianco AJ. 2011. Notch signalling in solid tumours: A little bit of everything but not all the time. Nat Rev Cancer 11: 338-351.

Reynolds BA, Weiss S. 1996. Clonal and population analyses demonstrate that an EGF-responsive mammalian embryonic CNS precursor is a stem cell. Dev Biol 175: 1-13.

Ripani E, Sacchetti A, Corda D, Alberti S. 1998. Human Trop-2 is a tumor-associated calcium signal transducer. Int I Cancer 76: $671-676$.
Salm SN, Koikawa Y, Ogilvie V, Tsujimura A, Coetzee S, Moscatelli D, Moore E, Lepor H, Shapiro E, Sun TT, et al. 2000. Generation of active TGF- $\beta$ by prostatic cell cocultures using novel basal and luminal prostatic epithelial cell lines. J Cell Physiol 184: 70-79.

Schroeter EH, Kisslinger JA, Kopan R. 1998. Notch-1 signalling requires ligand-induced proteolytic release of intracellular domain. Nature 393: 382-386.

Shahi P, Seethammagari MR, Valdez JM, Xin L, Spencer DM. 2011. Wnt and Notch pathways have interrelated opposing roles on prostate progenitor cell proliferation and differentiation. Stem Cells 29: 678-688.

Shih LB, Xuan H, Aninipot R, Stein R, Goldenberg DM. 1995. In vitro and in vivo reactivity of an internalizing antibody, RS7, with human breast cancer. Cancer Res 55: 5857s-5863s.

Shou J, Ross S, Koeppen H, de Sauvage FJ, Gao WQ. 2001. Dynamics of notch expression during murine prostate development and tumorigenesis. Cancer Res 61: 7291-7297.

Solanas G, Cortina C, Sevillano M, Batlle E. 2011. Cleavage of E-cadherin by ADAM10 mediates epithelial cell sorting downstream of EphB signalling. Nat Cell Biol 13: 1100-1 107.

Stein R, Chen S, Haim S, Goldenberg DM. 1997. Advantage of yttrium-90-labeled over iodine-131-labeled monoclonal antibodies in the treatment of a human lung carcinoma xenograft. Cancer 80: 2636-2641.

Trerotola M, Cantanelli P, Guerra E, Tripaldi R, Aloisi AL, Bonasera V, Lattanzio R, Lange RD, Weidle UH, Piantelli M, et al. 2012a. Upregulation of Trop-2 quantitatively stimulates human cancer growth. Oncogene (in press). doi: 10.1038/onc.2012.36.

Trerotola M, Li J, Alberti S, Languino LR. 2012b. Trop-2 inhibits prostate cancer cell adhesion to fibronectin through the $\beta 1$ integrin-RACK1 axis. J Cell Physiol 227: 3670-3677.

Tsukahara Y, Tanaka M, Miyajima A. 2011. TROP2 expressed in the trunk of the ureteric duct regulates branching morphogenesis during kidney development. PLOS ONE 6: e28607. doi: 10.1371 /journal.pone.0028607.

van Leenders GJLH, Dukers D, Hessels D, van den Kieboom SWM, Hulsbergen CA, Witjes JA, Otte AP, Meijer CJ, Raaphorst FM. 2007. Polycomb-group oncogenes EZH2, BMI1, and RING1 are overexpressed in prostate cancer with adverse pathologic and clinical features. Eur Urol 52: 455463.

Visvader JE. 2011. Cells of origin in cancer. Nature 469: 314322.

Wang XD, Leow CC, Zha J, Tang Z, Modrusan Z, Radtke F, Aguet M, de Sauvage FJ, Gao WQ. 2006. Notch signaling is required for normal prostatic epithelial cell proliferation and differentiation. Dev Biol 290: 66-80.

Wang J, Day R, Dong Y, Weintraub SJ, Michel L. 2008. Identification of Trop-2 as an oncogene and an attractive therapeutic target in colon cancers. Mol Cancer Ther 7: 280285.

Wang J, Zhang K, Grabowska D, Li A, Dong Y, Day R, Humphrey P, Lewis J, Kladney RD, Arbeit JM, et al. 2011. Loss of Trop2 promotes carcinogenesis and features of epithelial to mesenchymal transition in squamous cell carcinoma. Mol Cancer Res 9: 1686-1695.

Weng AP, Ferrando AA, Lee W, Morris JP IV, Silverman LB, Sanchez-Irizarry C, Blacklow SC, Look AT, Aster JC. 2004. Activating mutations of NOTCH1 in human T cell acute lymphoblastic leukemia. Science 306: 269-271.

Xin L, Ide H, Kim Y, Dubey P, Witte ON. 2003. In vivo regeneration of murine prostate from dissociated cell populations of postnatal epithelia and urogenital sinus mesenchyme. Proc Natl Acad Sci 100: 11896-11903. 
Xin L, Teitell MA, Lawson DA, Kwon A, Mellinghoff IK, Witte ON. 2006. Progression of prostate cancer by synergy of AKT with genotropic and nongenotropic actions of the androgen receptor. Proc Natl Acad Sci 103: 7789-7794.

Xin L, Lukacs RU, Lawson DA, Cheng D, Witte ON. 2007. Selfrenewal and multilineage differentiation in vitro from murine prostate stem cells. Stem Cells 25: 2760-2769.

Yardy GW, Brewster SF. 2005. Wnt signalling and prostate cancer. Prostate Cancer Prostatic Dis 8: 119-126.

Yardy GW, Bicknell DC, Wilding JL, Bartlett S, Liu Y, Winney B, Turner GD, Brewster SF, Bodmer WF. 2009. Mutations in the AXIN1 gene in advanced prostate cancer. Eur Urol 56: 486494.

Yoshimoto M, Cutz JC, Nuin PA, Joshua AM, Bayani J, Evans AJ, Zielenska M, Squire JA. 2006. Interphase FISH analysis of PTEN in histologic sections shows genomic deletions in $68 \%$ of primary prostate cancer and $23 \%$ of high-grade prostatic intra-epithelial neoplasias. Cancer Genet Cytogenet 169: 128-137.

Young DSF, Findlay HP, Hahn SE, DaCruz LAG, Ferry AL. 2008. Cytotoxicity mediation of cells evidencing surface expression of TROP-2. U.S. Patent US 7420041 B2.

Zhao G, Liu Z, Ilagan MX, Kopan R. 2010. $\gamma$-Secretase composed of PS1/Pen2/Aph1a can cleave notch and amyloid precursor protein in the absence of nicastrin. J Neurosci 30: 1648-1656. 


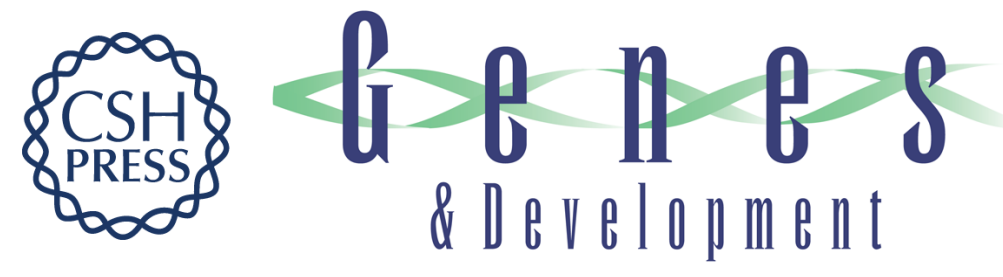

\section{Regulated proteolysis of Trop2 drives epithelial hyperplasia and stem cell self-renewal via $\beta$-catenin signaling}

Tanya Stoyanova, Andrew S. Goldstein, Houjian Cai, et al.

Genes Dev. 2012, 26:

Access the most recent version at doi:10.1101/gad.196451.112

Supplemental
Material http://genesdev.cshlp.org/content/suppl/2012/11/02/26.20.2271.DC1

References This article cites 62 articles, 23 of which can be accessed free at: http://genesdev.cshlp.org/content/26/20/2271.full.html\#ref-list-1

License Freely available online through the Genes \& Development Open Access option.

Email Alerting Receive free email alerts when new articles cite this article - sign up in the box at the top Service right corner of the article or click here.

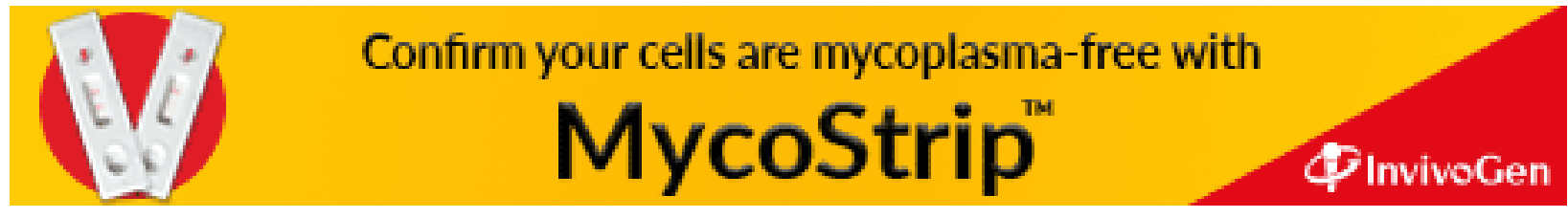

